\title{
Ovarian Tissue Cryopreservation as a Fertility Sparing Method in Oncological Patients-Current State and Controversy
}

\author{
Pankiewicz K ${ }^{1 *}$, Szewczyk $G^{1,2}$ and Maciejewski TM ${ }^{1}$ \\ ${ }^{1}$ Department of Obstetrics and Gynecology, Institute of Mother and Child, Warsaw, Poland \\ ${ }^{2}$ Department of General and Experimental Pathology, Warsaw Medical University, Poland \\ *Corresponding author: Pankiewicz K, Department of Obstetrics and Gynecology, Institute of Mother and Child, Warsaw, Poland
}

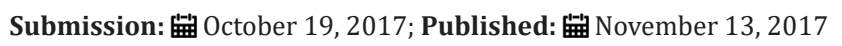

\begin{abstract}
Ovarian tissue cryopreservation is one of the possibilities in fertility preservation strategies dedicated to patients undergoing oncological treatment still found as experimental. Authors of this mini review discuss its usefulness with all substantial disadvantages that arouses controversy.
\end{abstract}

Keywords: Ovarian tissue cryopreservation; Fertility; Oncological treatment

\section{Introduction}

The number of men and women suffering from cancer is still growing. Increasing cancer morbidity among children, adolescents and young adults force the researchers to development of successful fertility sparing methods. One of them, however still found as experimental, is ovarian tissue cryopreservation.

\section{The impact of cancer on fertility}

Oncological treatment, both chemo- and radiotherapy, has a negative influence on reproductive and endocrine functions and is related to a high risk of premature ovarian insufficiency (POI). The use of chemotherapy may cause increased follicular apoptosis, ovarian cortical fibrosis, damage to ovarian vasculature and premature activation, recruitment and destruction of follicles. However, this effect depends strongly on patient's age, drug type and dose administered in treatment [1,2]. Radiotherapy has an influence not only on the ovaries but also on the uterus and the hypothalamic-pituitary-gonadal axis. Its use is related not only with POI but also with higher rates of miscarriage, preterm labor and fetal growth restriction $[3,4]$.

\section{Fertility preservation strategies}

According to the American Society of Reproductive Medicine recommendations, fertility-sparing methods can be divided into standard and experimental [5]. To the standard methods belong: gonadal shielding and ovarian transposition for radiotherapy, embryo and oocyte cryopreservation for other treatment opions. Experimental methods include: ovarian tissue cryopreservation
(OTC) and transplantation, oocyte in vitro maturation (IVM) and hormonal ovarian suppression with the use of gonadotropinreleasing hormone (GnRH) analogs.

\section{Ovarian tissue cryopreservation}

This method involves the removal of ovarian cortical tissue during laparoscopic or classic surgery. Obtained tissue is then cryo preserved and after the completion of oncological treatment is then auto transplanted in two possible locations: ortho topic, (which includes ovarian fossa or pelvic cavity) and hetero topic (which includes forearm or abdominal wall). The first ovarian tissue transplantation was performed in 1999 with the first pregnancy not being reported until 2004 [6-10]. The first pregnancy was reported in 2004 in patient treated with both chemotherapy and radiation for clinical stage IV Hodgkin lymphoma [11]. Until 2017 there were only 86 live births after orthotopic transplantations and no live births after hetero topic transplantation reported $[12,13]$. Moreover, the number of pregnancies after ovarian tissue transplantation can be confounded by the possibility of ovulation of oocytes from the native ovary and not the transplanted tissue.

OTC can be used individually or together with other methods (for e.g. ovarian transposition, IVM or oocyte cryopreservation) in the same patient to achieve the highest efficiency of fertility sparing. Ovarian tissue cryo preservation can be very useful in patients requiring immediate gonadotoxic treatment of aggressive neoplasm, when there is no time for ovarian stimulation, oocyte retrieval and cryopreservation of oocytes or embryos. It is also 
the only possibility for fertility preservation in prepubertal girls [14]. Cryopreservation of ovarian tissue includes two methods: slow freezing or vitrification. Slow freezing is a classic method of cryopreservation whereas vitrification is a new, rapid method of cryopreservation developed to eliminate the risk of ice formation in ovarian tissue. Till now there are no pregnancies reported after vitrification of ovarian tissue. However, the results of oocyte and embryos vitrification are very satisfying and that allows to predict the possible high efficiency $[15,16]$.

The advantages of ovarian tissue cryopreservation are that patient does not require ovarian stimulation and there is also no need for having a partner or using a sperm donor. Disadvantages include the risk of surgical and anesthetic procedures (necessary for removing and transplantation of ovarian tissue) and the risk of reseeding cancer in the future following auto transplantation. The American Society for Reproductive Medicine has reported the highest risk for ovarian metastasis in leukemia's and neuroblastomas [5]. Although, to date, there is no case of ovarian tissue exposing a patient to malignancy after transplantation, this risk must be taken into consideration in such patients.

Rosendahl et al. [17] Presented a study in which they examined ovarian tissue retrieved for cryopreservation using polymerase chain reaction to detect leukemic infiltration. They found that $75 \%$ of tissue samples showed leukemic infiltration, contrary to $0 \%$ rate of invasion on standard histologic examination [17]. It is now evidenced that for leukemic patients obtaining ovarian tissue after the first remission and before bone marrow transplantation may significantly decrease the risk of transmission from re implanted tissue. In a systematic review of auto transplantation of ovarian tissue from 289 patients with different malignancies (such as leukemia, lymphoma, sarcoma, breast, colorectal and gastric cancers) metastases were less common in most cancers, except for leukemia [18].

The future direction in development of described fertility preservation method seems to be ovarian tissue cryopreservation followed by in vitro maturation of oocytes retrieved from ovarian tissue and in vitro fertilization with embryo transfer. This procedure allows avoiding many disadvantages, such as the risk of metastasis after ovarian tissue auto transplantation and the risk of recurrence in patients with estrogen-dependent cancer (most of all breast cancer) during controlled ovarian stimulation protocols before classic oocyte retrieval. Moreover, it gives patients with hereditary cancer predisposition (for e.g. BRCA mutations) a possibility of preimplantation genetic diagnostics (PGD) in order to select embryos without familial cancer-predisposing mutations [19].

\section{Summary}

Increasing numbers of cancer survivors in the group of children and adolescents face the challenge of ameliorating the patient's quality of life. Cancer treatment, both chemo and radiotherapy have been shown to impair the whole reproductive axis and may lead to infertility. Therefore, there is a great need to develop methods for fertility preservation and ovarian tissue cryopreservation is one of them. Despite the many advantages of this method, it still becomes experimental and arouses controversy.

\section{References}

1. Ronn R, Holzer HEG (2013) Oncofertility in Canada: the impact of cancer on fertility. Curr Oncol 20(4): e338-e344.

2. Goodwin PJ, Ennis M, Pritchard KI, Trudeau M, Hood N, et al. (1999) Risk of menopause during the first year after breast cancer diagnosis. J Clin Oncol 17(8): 2365-2370.

3. Wo JY, Viswanathan AN (2009) Impact of radiotherapy on fertility, pregnancy, and neonatal outcomes in female cancer patients. Int J Radiat Oncol Biol Phys 73(5): 1304-1312.

4. Hawkins MM, Smith RA (1989) Pregnancy outcomes in childhood cancer survivors: probable effects of abdominal irradiation. Int J Cancer 43(3): 399-402.

5. Practice Committee of American Society for Reproductive Medicine (2013) Fertility preservation in patients undergoing gonadotoxic therapy or gonadectomy: a committee opinion. Fertil Steril 100(5): 1214-1223.

6. Jakimiuk AJ, Fritz A (2005) Ovarian tissue cryopreservation as a perspective for fertility preservation. Endokrynol Pol 56(6): 998-1001.

7. Oktay K, Buyuk E, Veeck L, Zaninovic N, Xu K, et al. (2004) Embryo development after heterotopic transplantation of cryopreserved ovarian tissue. Lancet 363(9412): 837-840.

8. Oktay K, Economos K, Kan M, Rucinski J, Veeck L, et al. (2001) Endocrine function and oocyte retrieval after autologous transplantation of ovarian cortical strips to the forearm. JAMA 286(12): 1490-1493.

9. Oktay K, Karlikaya G (2000) Ovarian function after transplantation of frozen, banked autologous ovarian tissue. N Engl J Med 342(22): 1919.

10. Oktay K, Bedoschi G, Pacheco F, Turan V, Emirdar V (2016) First pregnancies, live birth, and in vitro fertilization outcomes after transplantation of frozen-banked ovarian tissue with a human extracellular matrix scaffold using robot-assisted minimally invasive surgery. Am J Obstet Gynecol 214(1): 94.e1-99.e1.

11. Donnez J, Dolmans MM, Demylle D (2004) Livebirth after orthotopic transplantation of cryopreserved ovarian tissue. The Lancet 364(9443): 1405-1410.

12. Donnez J, Dolmans MM, Pellicer A, Diaz GC, Sanchez SM, et al. (2013) Restoration of ovarian activity and pregnancy after transplantation of cryopreserved ovarian tissue: a review of 60 cases of reimplantation. Fertil Steril 99(6): 1503-1513.

13. Oktay K, Türkçüoğlu I, Rodriguez WKA (2011) Four spontaneous pregnancies and three live births following subcutaneous transplantation of frozen banked ovarian tissue: what is the explanation? Fertil Steril 95(2): 804.e7-810.e7.

14. Pankiewicz K, Szewczyk G, Maciejewski TM, Szukiewicz D (2017) Strategies for overcoming oncological treatment-related ovarian dysfunction - literature review. Gynecol Endocrinol 33(11):830-835.

15. Edgar DH, Gook DA (2012) A critical appraisal of cryopreservation (slow cooling versus vitrification) of human oocytes and embryos. Hum Reprod Update 18(5): 536-540.

16. Dalman A, Deheshkar GFNS, Totonchi M, Pirjani R, Ebrahimi B, et al. (2017) Slow freezing versus vitrification technique for human ovarian tissue cryopreservation: An evaluation of histological changes, WNT signaling pathway and apoptotic genes expression. Cryobiology 2240(17): 30312-30317.

17. Rosendahl M, Andersen MT, Ralfkiær E, Kjeldsen L, Andersen MK, et al. (2010) Evidence of residual disease in cryopreserved ovarian cortex from female patients with leukemia. Fertil Steril 94(6): 2186-2190. 
18. Bastings L, Beerendonk CC, Westphal JR, Massuger LF, Kaal SE, et al. (2013) Autotransplantation of cryopreserved ovarian tissue in cancer survivors and the risk of reintroducing malignancy: a systematic review. Hum Reprod Update 19(5):483-506.
19. Konstantopoulou I, Pertesi M, Fostira F, Grivas A, Yannoukakos D, et al. (2009) Hereditary cancer predisposition syndromes and preimplantation genetic diagnosis: where are we now? J BUON 14 (Suppl 1): S187-S192. 